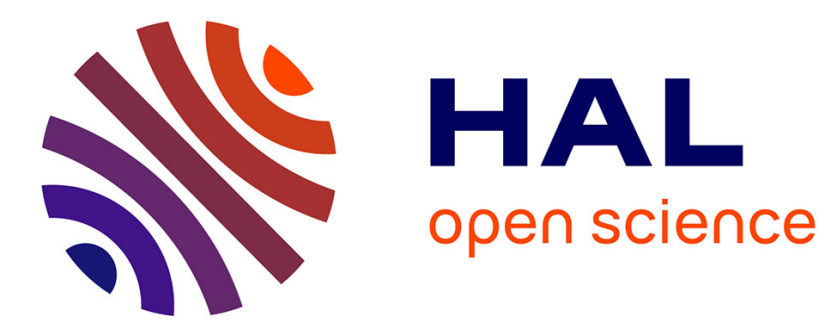

\title{
Quelques Points Importants en Détonique Classique
}

F. Chaisse

\section{To cite this version:}

F. Chaisse. Quelques Points Importants en Détonique Classique. Journal de Physique IV Proceedings, 1995, 05 (C4), pp.C4-57-C4-58. 10.1051/jp4:1995405 . jpa-00253704

\section{HAL Id: jpa-00253704 https://hal.science/jpa-00253704}

Submitted on 1 Jan 1995

HAL is a multi-disciplinary open access archive for the deposit and dissemination of scientific research documents, whether they are published or not. The documents may come from teaching and research institutions in France or abroad, or from public or private research centers.
L'archive ouverte pluridisciplinaire HAL, est destinée au dépôt et à la diffusion de documents scientifiques de niveau recherche, publiés ou non, émanant des établissements d'enseignement et de recherche français ou étrangers, des laboratoires publics ou privés. 


\title{
Quelques Points Importants en Détonique Classique
}

\author{
F. Chaisse
}

CEA/CEV-M, BP. 7, 77181 Courtry, France

\begin{abstract}
In this discussion we recall two types of experiments, achieved at the CEV-M (CEA), exhibiting specific properties in the detonation dynamics.

In the classical theory of detonation (ZND scheme) it appears some troubles in the interpretation of such experimental results.
\end{abstract}

Dans le cadre de la détonique classique, des études expérimentales conduites au CEV-M ces dernières années ont fait apparaître des propriétés spécifiques de la dynamique des ondes de détonation qui semblent, à première vue, difficilement explicables dans le cadre du modèle ZND.

Pour cela, nous nous référons aux publications [1] et [2] de MM. J. Aveillé et J.M. Chevalier. Le premier a étudié la détonation sphérique divergente sur deux compositions explosives au TATB (T1 et T2) et sur une composition à l'octogène (X1), le second sur le même type de compositions explosives a mis en évidence l'existence d'onde de front se propageant transversalement sur la surface de l'onde de détonation en symétrie cylindrique.

Dans la publication [1], sur les trois compositions étudiées, on constate que, pour une courbure donnée du front, la célérité du front de détonation en sphérique divergent reste toujours inférieure à celle de la détonation axi-symétrique. La concordance de ces célérités de détonation n'est observée qu'aux faibles courbures.

Dans la publication [2], la célérité $C$ de propagation d'onde de front, associée à une perturbation prenant naissance sur le contour externe de la cartouche cylindrique, possède un caractère fini. Cette célérité $\mathrm{C}$ présente la propriété de croître lorsque la célérité axiale du front de détonation diminue.

Une question alors se pose : comment interpréter les résultats des références [1] en [2] d'un point de vue théorique?

Si l'on se réfère au code DSD (Detonation Shock Dynamics) développé à LOS ALAMOS [3] qui détermine l'évolution spatio-temporelle d'un front d'onde de détonation sans faire appel à 
l'écoulement aval, nous avons des difficultés théoriques. Dans le code DSD, la détonation possède la structure usuelle ZND associée à l'existence d'une relation célérité-courbure, et le traitement théorique de ce modèle conduit à une équation d'évolution du front d'onde de type parabolique.

\section{Références :}

[1] J. Aveillé and al.

Experimental Study of Spherically Diverging Detonation Waves. 8th Symp. Int. on Detonation - Albuquerque (N.M.) (1985).

[2] J.M. Chevalier and al.

Propagation Phenomena on the Detonation Wave Front.

Physical Review Letters - Vol. $71-n^{\circ} 5$ - p. $712-714$ (1993).

[3] J. Bdzil and W. Fickett.

DSD Technology : A Detonation Reactive Huygens Code.

L.A.N.L. rapport LA.12235.MS (1992). 